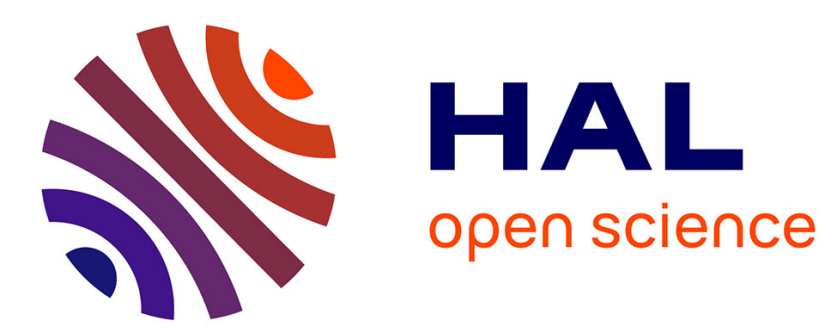

\title{
Fourth-order convergence of a compact scheme for the one-dimensional biharmonic equation
}

\author{
D Fishelov, M Ben-Artzi, J-P Croisille
}

\section{To cite this version:}

D Fishelov, M Ben-Artzi, J-P Croisille. Fourth-order convergence of a compact scheme for the onedimensional biharmonic equation. ICNAAM 2012 10th International Conference of Numerical Analysis and Applied Mathematics, Sep 2012, Kos, Greece. pp.1101-1104, 10.1063/1.4756339 . hal-01283671

\section{HAL Id: hal-01283671 \\ https://hal.science/hal-01283671}

Submitted on 8 Mar 2016

HAL is a multi-disciplinary open access archive for the deposit and dissemination of scientific research documents, whether they are published or not. The documents may come from teaching and research institutions in France or abroad, or from public or private research centers.
L'archive ouverte pluridisciplinaire HAL, est destinée au dépôt et à la diffusion de documents scientifiques de niveau recherche, publiés ou non, émanant des établissements d'enseignement et de recherche français ou étrangers, des laboratoires publics ou privés. 


\title{
FOURTH-ORDER CONVERGENCE OF A COMPACT SCHEME FOR THE ONE-DIMENSIONAL BIHARMONIC EQUATION
}

\author{
D. FISHELOV ${ }^{1}$ M. BEN-ARTZI ${ }^{2}$ J-P. CROISILLE ${ }^{3}$ \\ ${ }^{1}$ AFEKA - TEL-AVIV ACADEMIC COLLEGE OF ENGINEERING, \\ EMAIL:DALIAF@POST.TAU.AC.IL \\ ${ }^{2}$ THE HEBREW UNIVERSITY,ISRAEL \\ ${ }^{3}$ UNIVERSITY OF PAUL VERLAINE-METZ, FRANCE
}

\begin{abstract}
The convergence of a fourth-order compact scheme to the one-dimensional biharmonic problem is established in the case of general Dirichlet boundary conditions. The compact scheme invokes value of the unknown function as well as Pade approximations of its first-order derivative. Using the Pade approximation allows us to approximate the first-order derivative within fourth-order accuracy. However, although the truncation error of the discrete biharmonic scheme is of fourth-order at interior point, the truncation error drops to first-order at near-boundary points. Nonetheless, we prove that the scheme retains its fourth-order (optimal) accuracy. This is done by a careful inspection of the matrix elements of the discrete biharmonic operator. A number of numerical examples corroborate this effect.

We also present a study of the eigenvalue problem $u_{x x x x}=\nu u$. We compute and display the eigenvalues and the eigenfunctions related to the continuous and the discrete problems. By the positivity of the eigenvalues, one can deduce the stability of of the related time-dependent problem $u_{t}=-u_{x x x x}$. In addition, we study the eigenvalue problem $u_{x x x x}=\nu u_{x x}$. This is related to the stability of the linear time-dependent equation $u_{x x t}=\nu u_{x x x x}$. Its continuous and discrete eigenvalues and eigenfunction (or eigenvectors) are computed and displayed graphically.
\end{abstract}

\section{Derivation of THREE-POINT COMPACT OPERATORS}

We consider here the one-dimensional biharmonic equation on the interval $[a, b]$. For the simplicity of the presentation, we choose homogeneous boundary conditions. The one-dimensional biharmonic equation is

$$
\left\{\begin{array}{l}
u^{(4)}(x)=f(x), \quad a<x<b \\
u(a)=0, u(b)=0, u^{\prime}(a)=0, u^{\prime}(b)=0
\end{array}\right.
$$

We look for a high-order compact approximation to (1). We lay out a uniform grid $a=x_{0}<x_{1}<\ldots<x_{N-1}<$ $x_{N}=b$. Here $x_{i}=i h$ for $0 \leq i \leq N$ and $h=(b-a) / N$.

In what follows, we shall use the notion of grid functions. A grid function is a function defined on the discrete grid $\left\{x_{i}\right\}_{i=0}^{N}$. We denote grid functions with fraktur letters such as $\mathfrak{u}, \mathfrak{v}$. We have

$$
\mathfrak{u}=\left(\mathfrak{u}\left(x_{0}\right), \mathfrak{u}\left(x_{1}\right), \cdots, \mathfrak{u}\left(x_{N-1}\right), \mathfrak{u}\left(x_{N}\right)\right) .
$$

In addition, we denote by $u^{*}=\left(u\left(x_{0}\right), u\left(x_{1}\right), \cdots, u\left(x_{N-1}\right), u\left(x_{N}\right)\right)$ the grid function, which consists of the values of $u(x)$ at grid points.

We denote by $l_{h}^{2}$ the functional space of grid functions. This space is equipped with a scalar product and an associated norm

$$
(\mathfrak{u}, \mathfrak{v})_{h}=h \sum_{i=0}^{N} \mathfrak{u}\left(x_{i}\right) \mathfrak{v}\left(x_{i}\right), \quad|\mathfrak{u}|_{h}=(\mathfrak{u}, \mathfrak{u})_{h}^{1 / 2} .
$$

We also define the sup norm for a grid function $\mathfrak{u}|\mathfrak{u}|_{\infty}=\max _{0 \leq i \leq N}\left|\mathfrak{u}\left(x_{i}\right)\right|$.

We define the difference operators $\delta_{x}, \delta_{x}^{2}$ on grid functions by

$$
\delta_{x} \mathfrak{u}_{i}=\frac{\mathfrak{u}_{i+1}-\mathfrak{u}_{i-1}}{2 h}, \quad \delta_{x}^{2} \mathfrak{u}_{i}=\frac{\mathfrak{u}_{i+1}-2 \mathfrak{u}_{i}+\mathfrak{u}_{i-1}}{h^{2}}, \quad 1 \leq i \leq N-1 .
$$

Date: July 23, 2012

* Partially supported by a French-Israeli scientific cooperation grant 3-1355. The first author was also supported by a research grant of Afeka-Tel-Aviv Academic College of Engineering. 
In these definitions the boundary values $\mathfrak{u}_{0}, \mathfrak{u}_{N}$ are assumed to be known.

Suppose that we are given data $u_{i-1}^{*}, u_{i}^{*}, u_{i+1}^{*}$ at the grid points $x_{i-1}, x_{i}, x_{i+1}$. In addition, we are given some approximations $u_{x, i-1}^{*}, u_{x, i+1}^{*}$ for $u^{\prime}\left(x_{i-1}\right), u^{\prime}\left(x_{i+1}\right)$. We seek a polynomial of degree 4

$$
p(x)=u_{i}^{*}+a_{1}\left(x-x_{i}\right)+a_{2}\left(x-x_{i}\right)^{2}+a_{3}\left(x-x_{i}\right)^{3}+a_{4}\left(x-x_{i}\right)^{4},
$$

which interpolates the data $u_{i-1}^{*}, u_{i}^{*}, u_{i+1}^{*}, u_{x, i-1}^{*}, u_{x, i+1}^{*}$.

The coefficients $a_{1}, a_{2}, a_{3}, a_{4}$ of the polynomial are

$$
\left\{\begin{array}{l}
a_{1}=\frac{3}{4 h}\left(u_{i+1}^{*}-u_{i-1}^{*}\right)-\left(\frac{1}{4} u_{x, i+1}^{*}+\frac{1}{4} u_{x, i-1}^{*}\right), \\
a_{2}=\frac{1}{h^{2}}\left(u_{i+1}^{*}+u_{i-1}^{*}-2 u_{i}^{*}\right)-\frac{1}{4 h}\left(u_{x, i+1}^{*}-u_{x, i-1}^{*}\right)=\delta_{x}^{2} u_{i}^{*}-\frac{1}{2}\left(\delta_{x} u_{x}^{*}\right)_{i}, \\
a_{3}=-\frac{1}{4 h^{3}}\left(u_{i+1}^{*}-u_{i-1}^{*}\right)+\frac{1}{4 h^{2}}\left(u_{x, i+1}^{*}+u_{x, i-1}^{*}\right), \\
a_{4}=-\frac{1}{2 h^{4}}\left(u_{i+1}^{*}+u_{i-1}^{*}-2 u_{i}^{*}\right)+\frac{1}{4 h^{3}}\left(u_{x, i+1}^{*}-u_{x, i-1}^{*}\right)=\frac{1}{2 h^{2}}\left(\left(\delta_{x} u_{x}^{*}\right)_{i}-\delta_{x}^{2} u_{i}^{*}\right) .
\end{array}\right.
$$

The coefficients above require the data $u_{i}^{*}$ and $u_{x, i}^{*}$. In the case where only the values of $u_{i}^{*}$ are given, then $\left\{u_{x, i}^{*}\right\}_{i=1}^{N-1}$ have to be evaluated in terms of $\left\{u_{i}^{*}\right\}_{i=0}^{N}$. Looking at the first equation in (6), we see that a natural candidate for $u_{x, i}^{*}$ is $u_{x, i}^{*}=a_{1}$.

This yields

$$
\frac{1}{6} u_{x, i}^{*}+\frac{2}{3} u_{x, i}^{*}+\frac{1}{6} u_{x, i+1}^{*}=\delta_{x} u_{i}^{*} .
$$

This is by definition the Hermitian derivative. If we introduce the three-point operator $\sigma_{x}$ on grid functions by

$$
\sigma_{x} \mathfrak{v}_{i}=\frac{1}{6} \mathfrak{v}_{i-1}+\frac{2}{3} \mathfrak{v}_{i}+\frac{1}{6} \mathfrak{v}_{i+1}, \quad 1 \leq i \leq N-1
$$

can rewrite $(7)$ as

$$
\sigma_{x} u_{x, i}^{*}=\delta_{x} u_{i}^{*}, \quad 1 \leq i \leq N-1
$$

This suggests that $\delta_{x}^{4} u_{i}^{*}$ is an approximation to the fourth-order derivative of $u$ at $x_{i}$, namely,

$$
\delta_{x}^{4} u_{i}^{*}=\frac{12}{h^{2}}\left(\left(\delta_{x} u_{x}^{*}\right)_{i}-\delta_{x}^{2} u_{i}^{*}\right) .
$$

This approximation, called the discrete biharmonic approximation (see also $[3,5]$ ). Note that, in the non-periodic setting, boundary values of $u_{x}$ should be given in order to compute $\delta_{x}^{4}$ at near boundary points $x_{1}, x_{N-1}$.

We refer to $\delta_{x}^{4}$ as the discrete biharmonic operator (DBO). We define

Definition 1 (Discrete biharmonic operator $(\mathrm{DBO}))$. Let $\mathfrak{u} \in l_{h}^{2}$ be a given grid function. The discrete biharmonic operator is defined by

$$
\delta_{x}^{4} \mathfrak{u}_{i}=\frac{12}{h^{2}}\left(\delta_{x} \mathfrak{u}_{x, i}-\delta_{x}^{2} \mathfrak{u}_{i}\right), \quad 1 \leq i \leq N-1 .
$$

Here $\mathfrak{u}_{x}$ is the Hermitian derivative of $\mathfrak{u}$ satisfying (9) with given boundary values $\mathfrak{u}_{x, 0}$ and $\mathfrak{u}_{x, N}$.

Using (10) and (7), the solution of (1) may be approximated by the scheme

$$
\left\{\begin{array}{l}
\text { (a) } \delta_{x}^{4} \mathfrak{u}_{i}=f\left(x_{i}\right) \quad 1 \leq i \leq N-1, \\
\text { (b) } \frac{1}{6} \mathfrak{u}_{x, i-1}+\frac{2}{3} \mathfrak{u}_{x, i}+\frac{1}{6} \mathfrak{u}_{x, i+1}=\delta_{x} \mathfrak{u}_{i}, \quad 1 \leq i \leq N-1, \\
\text { (c) } \mathfrak{u}_{0}=0, \mathfrak{u}_{N}=0, \mathfrak{u}_{x, 0}=0, \mathfrak{u}_{x, N}=0 .
\end{array}\right.
$$




\section{Optimal Rate of Convergence of the one-Dimensional Stephenson scheme}

In order to prove the fourth-order convergence of the scheme, we invoke the matrix representation fo the discrete biharmonic operator.

In [1] we carried out an error analysis based on the coercivity of $\delta_{x}^{4}$. The analysis presented there was based on an energy $\left(l^{2}\right)$ method and led to a "sub-optimal" convergence rate of $h^{\frac{3}{2}}$. In [2] we have improved this result by showing that the convergence rate is almost three (the error is bounded by $C h^{3} \log (|h|)$. Here we prove the optimal (fourth-order) convergence of the scheme. Let $u$ be the exact solution of (1) and let $\mathfrak{u}$ be its approximation by the Stephenson scheme (12). Let $u^{*}$ be the grid function corresponding to $u$. We consider the error between the approximated solution $\mathfrak{u}$ and the collocated exact solution $u^{*}$,

$$
\mathfrak{e}=\mathfrak{u}-u^{*},
$$

The grid function $u^{*}$ satisfies

$$
\delta_{x}^{4} u_{i}^{*}=f^{*}\left(x_{i}\right)+\mathfrak{r}_{i}, \quad 1 \leq i \leq N-1,
$$

where $\mathfrak{r}$ is by definition the truncation error.

The error $\mathfrak{e}=\mathfrak{u}-u^{*}$ satisfies

$$
\begin{aligned}
& \delta_{x}^{4} \mathfrak{e}_{i}=-\mathfrak{r}_{i}, \quad 1 \leq i \leq N-1, \\
& \mathfrak{e}_{0}=0, \mathfrak{e}_{N}=0, \mathfrak{e}_{x, 0}=0, \mathfrak{e}_{x, N}=0 .
\end{aligned}
$$

We prove the following error estimate.

Theorem 2. Let $u$ be the exact solution of (1) and assume that $u$ has continuous derivatives up to order eight on $[a, b]$. Let $\mathfrak{u}$ be the approximation to $u$, given by the Stephenson scheme (12). Let $u^{*}$ be the grid function corresponding to $u$. The, the error $\mathfrak{e}=\mathfrak{u}-u^{*}$ satisfies

$$
|\mathfrak{e}|_{h} \leq C h^{4}
$$

where $C$ depends only on $f$.

\section{EIGENFUnCTIONS AND EIGENVALUES OF FOURTH-ORDER OPERATORS}

3.1. Eigenfunction Approach for $u_{x x x x}=\nu u$. Consider the eigenvalue problem for the one-dimensional biharmonic problem.

$$
\phi^{(4)}=\lambda \phi, \quad \phi(0)=\phi^{\prime}(0)=\phi(1)=\phi^{\prime}(1)=0 .
$$

It may be easily checked that the eigenfunctions for this problem are

$$
\begin{aligned}
& \phi_{k}(x)=\left(\sin \omega_{k}-\sinh \omega_{k}\right)\left(\cos \left(\omega_{k} x\right)-\cosh \left(\omega_{k} x\right)\right) \\
& \quad-\left(\cos \omega_{k}-\cosh \omega_{k}\right)\left(\sin \left(\omega_{k} x\right)-\sinh \left(\omega_{k} x\right)\right) \quad x \in[0,1], \quad k=1,2, \ldots,
\end{aligned}
$$

and

$$
\lambda_{k}=\omega_{k}^{4}, \quad k=1,2, \ldots,
$$

where $\omega_{k}$ satisfy

$$
\cos \left(\omega_{k}\right) \cosh \left(\omega_{k}\right)=1, \quad k=1,2, . ., \quad \omega_{k}>0 .
$$

Therefore, the eigenfunction of the continuous problem (16) have the following form

$$
\phi(x)=A \cos \left(\omega_{1} x\right)+B \sin \left(\omega_{1} x\right)+C \cosh \left(\omega_{2} x\right)+D \sinh \left(\omega_{2} x\right), \quad x \in[0,1],
$$

where $A, B, C, D \in \mathbb{R}$ and $\omega_{1}, \omega_{2}>0$.

We consider now the discrete problem. Let $\mathfrak{v}$ be the solution of the discrete biharmonic problem.

$$
\delta_{x}^{4} \mathfrak{v}=\lambda \mathfrak{v}, \quad \mathfrak{v}(0)=\mathfrak{v}_{x}(0)=\mathfrak{v}(1)=\mathfrak{v}_{x}(1)=0
$$

where $\mathfrak{v}_{x}$ is the Hermitian derivative of $\mathfrak{v}$. 
Claim 3. A complete set of $(N-1)$ linearly independent eigenfunctions (in $l_{h, 0}^{2}$ ) for problem (21) is given by

$$
\begin{aligned}
\mathfrak{v}_{i}^{(k)} & =\left(\sin \left(\omega_{1}^{(k)}\right)-T_{k} \sinh \left(\omega_{2}^{(k)}\right)\right) \cdot\left(\cos \left(\omega_{1}^{(k)} x_{i}\right)-\cosh \left(\omega_{2}^{(k)} x_{i}\right)\right) \\
& -\left(\cos \left(\omega_{1}^{(k)}\right)-\cosh \left(\omega_{2}^{(k)}\right)\right)\left(\sin \left(\omega_{1}^{(k)} x_{i}\right)-T_{k} \sinh \left(\omega_{2}^{(k)} x_{i}\right)\right), \quad k=1, . ., N-1,
\end{aligned}
$$

where

$$
T_{k}=\frac{\sinh ^{3}\left(\omega_{2}^{(k)} h\right)}{\sin ^{3}\left(\omega_{1}^{(k)} h\right)}
$$

and $\omega_{1}^{(k)}, \omega_{2}^{(k)}$ satisfy the following set of equations.

$$
\begin{gathered}
\frac{\sin ^{4}\left(\frac{\omega_{1}^{(k)} h}{2}\right)}{3-2 \sin ^{2}\left(\frac{\omega_{1}^{(k)} h}{2}\right)}=\frac{\sinh ^{4}\left(\frac{\omega_{2}^{(k)} h}{2}\right)}{3+2 \sinh ^{2}\left(\frac{\omega_{2}^{(k)} h}{2}\right)}, \\
2 \cos \left(\omega_{1}^{(k)}\right) \cosh \left(\omega_{2}^{(k)}\right)+R_{k} \sin \left(\omega_{1}^{(k)}\right) \sinh \left(\omega_{2}^{(k)}\right)=2, \quad R_{k}=T_{k}-\frac{1}{T_{k}} .
\end{gathered}
$$

The eigenvalues $\lambda_{k}$ are

$$
\lambda_{k}=\frac{48}{h^{4}} \frac{\sin ^{4}\left(\frac{\omega_{1}^{(k)} h}{2}\right)}{3-2 \sin ^{2} \frac{\omega_{1}^{(k)} h}{2}}, \quad k=1, \ldots, N-1 .
$$

3.2. Eigenfunction Approach for $u_{x x t}=\nu u_{x x x x}$. We consider a simple linear one-dimensional model for the Navier-Stokes equations in streamfunction formulation

$$
u_{x x t}=\nu u_{x x x x}
$$

where $u(x, t)$ is a function defined for $(x, t) \in[0,1] \times[0, \infty)$. Its steady-state solution satisfies the one-dimensional biharmonic problem, discussed in this paper. In addition, this equation serves as linear model for the streamfunction formulation of the Navier-Stokes system (see $[1,4]$ ). If $\psi$ is the streamfunction, then the Navier-Stokes system in streamfunction formulation takes the following form.

$$
\partial_{t}(\Delta \psi)+\left(\nabla^{\perp} \psi\right) \cdot \nabla(\Delta \psi)=\nu \Delta^{2} \psi
$$

It is evident that its restriction to the one-dimensional case with vanishing convective term is Equation (27).

We define an eigenfunction $\phi(x)$ to $(27)$ as a function satisfying, for some $\mu \in \mathbb{R}$ (which is the associated eigenvalue),

$$
\mu \phi^{\prime \prime}(x)=\phi^{(4)}(x), \quad x \in[0,1]
$$

along with the boundary conditions

$$
\phi(0)=\phi^{\prime}(0)=\phi(1)=\phi^{\prime}(1)=0 .
$$

Claim 4. A complete set of orthogonal eigenfunctions and their associate eigenvalues is given by the union of the following two families

$$
\left\{\begin{array}{c}
\phi_{k}^{(1)}(x)=1-\cos (2 \pi k x), \quad \mu_{k}^{(1)}=-(2 \pi k)^{2} \quad k=1,2, \ldots \\
\phi_{k}^{(2)}(x)=\frac{1}{\pi q_{k}} \sin \left(2 q_{k} \pi x\right)-\cos \left(2 q_{k} \pi x\right)-2 x+1, \quad \mu_{k}^{(2)}=-\left(2 \pi q_{k}\right)^{2}, \\
k=2,3, \ldots
\end{array}\right.
$$

where (for the second family), $q_{k}$ is the (unique) solution of

$$
\tan \left(q_{k} \pi\right)=q_{k} \pi, \quad q_{k} \in\left(k-1, k-\frac{1}{2}\right) .
$$




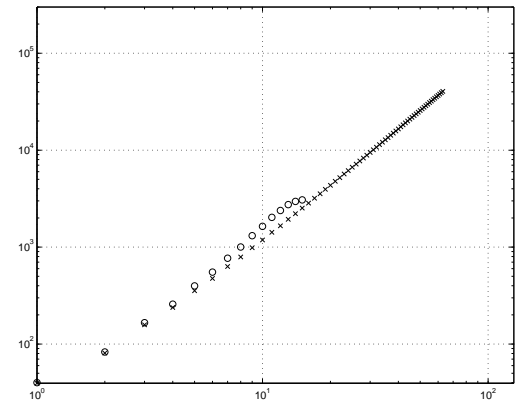

(a) Eigenvalues, $\mathrm{N}=16$

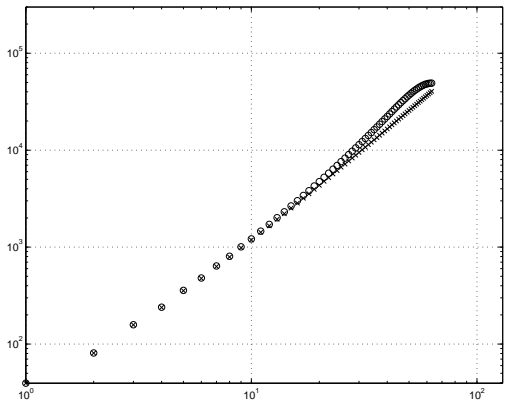

(b) Eigenvalues, $\mathrm{N}=64$

Figure 1. Spectral problem (29). Continuous ('x') versus discrete ('o') eigenvalues in Log-Log scale. Left panel: the discrete eigenvalues are computed with $N=16$. Right panel: the discrete eigenvalues are computed with $N=64$.

In the discrete problem the second-order and fourth-order spatial derivatives are replaced by their three-point counterparts, $\delta_{x}^{2}$ and $\delta_{x}^{4}$, respectively.

Specializing to the interval $[0,1]$ we use a uniform grid $\left\{x_{i}=i h, i=0,1, \ldots, N\right\}$.

Recall that for functions of the type

$$
v(x)=A \cos (a x)+B \sin (b x)+C x+D, \quad x \in[0,1], \quad A, B, C, D, a, b \in \mathbb{R},
$$

we compute the Hermitian derivative and the action of $\delta_{x}^{2}, \delta_{x}^{4}$ on $\mathfrak{v}$ and then we look for the discrete eigenvalues and eigenfunctions, namely, grid functions satisfying

$$
\mu \delta_{x}^{2} \mathfrak{v}=\delta_{x}^{4} \mathfrak{v}, \quad \mu \in \mathbb{R}
$$

subject to the homogeneous boundary conditions

$$
\mathfrak{v}\left(x_{0}\right)=\mathfrak{v}_{x}\left(x_{0}\right)=\mathfrak{v}\left(x_{N}\right)=\mathfrak{v}_{x}\left(x_{N}\right)=0 .
$$

In the following claim we show that the discrete eigenfunctions span the space $l_{h, 0}^{2}$. In fact, we give an explicit basis consisting of a union of two families of eigenfunctions, analogous to the continuous case of Claim 4 . The assumption that $N$ is even is made for notational simplicity.

Claim 5. Suppose that $N$ is even. Then a complete set of $(N-1)$ linearly independent eigenfunctions (in $\left.l_{h, 0}^{2}\right)$ is given by the union of the following two families

$$
\left\{\begin{array}{l}
\mathfrak{v}_{k}^{(1)}\left(x_{i}\right)=1-\cos \left(2 \pi k x_{i}\right), \quad 0 \leq i \leq N, \quad k=1,2, \ldots, \frac{N}{2}, \\
\mathfrak{v}_{k}^{(2)}\left(x_{i}\right)=A_{k} \sin \left(2 r_{k} \pi x_{i}\right)-\cos \left(2 r_{k} \pi x_{i}\right)-2 x_{i}+1, \quad 0 \leq i \leq N, \quad k=2, \ldots, \frac{N}{2},
\end{array}\right.
$$

where (for the second family), $A_{k}, r_{k}$ are uniquely determined by the pair of equations

$$
\frac{1}{A_{k}}=\tan \left(r_{k} \pi\right), \quad A_{k}=\frac{2 h}{3} \frac{2+\cos \left(2 r_{k} \pi h\right)}{\sin \left(2 r_{k} \pi h\right)}, \quad r_{k} \in\left(k-1, k-\frac{1}{2}\right) .
$$

Figure 1 displays the eigenvalues of Problem (29) versus the discrete spectrum for $N=16$ (left panel) and $N=64$ (right panel), using a Log-Log scale. Observe that the continuous spectrum is approximated by the discrete one from above.

\section{REFERENCES}

[1] M. Ben-Artzi, J-P. Croisille, and D. Fishelov. Convergence of a compact scheme for the pure streamfunction formulation of the unsteady Navier-Stokes system. SIAM J. Numer. Anal., 44,5:1997-2024, 2006.

[2] D. Fishelov, M. Ben-Artzi, and J-P. Croisille. Recent developments in the pure streamfunction formulation of the Navier-Stokes system. J. Sci. Comput., 45(1-3):238-258, 2010.

[3] M. M. Gupta, R.P.Manohar, and J.W.Stephenson. Single cell high order scheme for the convection-diffusion equation with variable coefficients. Int. J. Numer. Meth. Fluids, 4:641-651, 1984.

[4] R. Kupferman. A central-difference scheme for a pure streamfunction formulation of incompressible viscous flow. SIAM J. Sci. Comput., 23(1):1-18, 2001.

[5] J. W. Stephenson. Single cell discretizations of order two and four for biharmonic problems. J. Comput. Phys., 55:65-80, 1984. 